\title{
A Numerical Method for Computing Asymptotic States and Outgoing Distributions for Kinetic Linear Half-Space Problems
}

\author{
F. Golse ${ }^{1}$, A. Klar ${ }^{2}$
}

1 Université Paris 7, Denis Diderot, U.F.R. de Mathematiques, 2,place Jussieu, F-75251 Paris Cedex 05

2 Fachbereich Mathematik, Universität Kaiserslautern, Postfach 3049, D-67663 Kaiserslautern, E-Mail: klar@mathematik.uni-kl.de

Key words:

Domain Decomposition, Kinetic Linear Half Space Problem, Variational Methods

February 1995 


\title{
A Numerical Method for Computing Asymptotic States and Outgoing Distributions for Kinetic Linear Half-Space Problems
}

\author{
by FRANÇOIS GOLSE and AXEL KLAR
}

\begin{abstract}
Linear half space problems can be used to solve domain decomposition problems between Boltzmann and aerodynamic equations.

A new fast numerical method computing the asymptotic states and outgoing distributions for a linearized BGK half-space problem is presented. Relations with the so-called variational methods are discussed. In particular, we stress the connection between these methods and Chapman-Enskog type expansions.
\end{abstract}

\section{Introduction}

The Boltzmann equation and the more classical gas dynamics equations (such as Euler or Navier-Stokes equations) are used to model hypersonic gas flows. Numerical simulations of such flows are useful in the design of space vehicles, especially in understanding the behavior of the early phases of reentry flights.

Such flows are usually far from any kind of local equilibrium states: real gas effects (and the so many different degrees of freedom involved such as rotational and vibrational energies) as well as the importance of chemical reactions in the energy balance on the vehicle surface demand that variants of the Boltzmann equation be used as first principle equations instead of the Euler or Navier-Stokes equations. However, when the mean free path of molecules becomes small, all numerical methods for the Boltzmann equation become exceedingly espensive in computing time. Therefore, gas dynamics equations should be used whenever possible - in other words, near local equilibrium states in situations where the local mean free path is small and outside of shock and boundary layers. These considerations prompt the use of domain decomposition strategies, where the Boltzmann equation is to be solved only in regions others than those mentioned above. Once the regions described by the gas dynamics equations are determined, the next major problem is the matching of the Boltzmann domain with the Euler or Navier-Stokes domain. This question is far from being an easy one, as the equations to couple and the numerical schemes used to solve them are of very different nature. For numerical work on the coupling of Boltzmann- and gas dynamics equations, see (among other references) Bourgat et al. [3] , Lukschin et al. [16]. A more refined approach to find the correct coupling conditions is given for example by Illner and Neunzert [12]. 
The problem that we address in this article is to find as explicit as possible and yet accurate matching relations between the kinetic and gas dynamics regions. We confine our investigations to the case of an ideal gas, since our problem seems open even in this case. Yet, it is beyond doubt that some of the methods and ideas used here could be adapted to more realistic models.

As a general principle, the matching can be done by modelling the interface region by a transition layer where some "intermediate equation" (e.g., the linearized Boltzmann equation) is solved, see Golse [9] and Klar [14]. We assume this layer to have slab symmetry, that is, the particle distribution is constant on surfaces parallel to the interface. (This is generically the case whenever the curvature of the interface is small compared to the reciprocal of the mean free path). Hence, the space coordinate reduces to $x$, the distance to the interface. After scaling it like $\frac{x}{\epsilon}$, where $\epsilon$ is the order magnitude of the mean free path, one has to solve the following equation

$$
\begin{aligned}
\left(v_{1}+u\right) \partial_{x} \varphi+L \varphi & =0 \quad \text { with } \quad x \in[0, \infty) \quad \text { and } \quad v=\left(v_{1}, v_{2}, v_{3}\right) \in \mathbf{R}^{3} \\
\varphi(0, v) & =k(v), \quad v_{1}+u>0
\end{aligned}
$$

where $u$ is the component of the bulk velocity normal to the interface, $L$ the Boltzmann operator linearized around some local Maxwellian and $k$ the distribution function computed in the Boltzmann region. This problem should be solved at each interface "cell" or "mesh". A direct solution by any kind of iterative method seems much too expensive to do this. In fact, one is not really interested in the full solution: the only objects of interest are the asymptotic states, i.e. $\varphi(\infty, v)$ and the outgoing distribution $\varphi(0, v), v_{1}+u<0$. Indeed, the correct boundary conditions for the gas dynamics equations and the ingoing densities at the boundary of the Boltzmann region can be written in terms of those quantities only.

We shall describe in this paper a numerical procedure which computes just those quantities by using a Chapman-Enskog type expansion to approximate the solution. The method is seen to converge very fast numerically. It seems to give accurate results when compared to the available explicit solutions in some special cases and to results obtained by more direct simulation schemes. This numerical procedure is inspired by the work of Ringeisen [18], originally aimed at solving the one-speed transport equation with isotropic scattering with full line geometry; in this special case, Ringeisen was able to give a convergence proof for his method, while pointing out that it should be valid in more general contexts. The first step of our method in the special case $u=0$ is shown to be equivalent to the so-called variational methods developed by Cercignani [4], Golse [10] and Loyalka, Ferziger [15] and used for determining the slip boundary coefficients for the Navier Stokes equation. For other approaches to the numerical solution of the above half space problem we refer to Aoki, Sone [1], Coron [7], Siewert,Thomas [19] and for a mathematical investigation to Arthur, Cercignani [2], Cercignani [5], Coron et al. [8], Greenberg et al. [11] and Siewert, Thomas [19].

This paper is organized as follows:

In Chapter 2, where we explain the method for a 1-dimensional model equation, as well 
as in Chapter 3, where the 3-dimensional (in velocity) BGK-equation is considered, we proceed in the following systematic way:

Part 1 introduces the equations;

Part 2 describes the method for computing the asymptotic states and the "albedo operator" - see the definition below - for $u>0$;

Part 3 specializes to the case $u=0$ and compares the results with those of the variational method;

Part 4 discusses the numerical results.

\section{A 1-Dimensional Boltzmann Equation}

In this section we use a simple stationary 1-dimensional model Boltzmann equation to describe our numerical procedure. In Chapter 3 this procedure will be applied to the linearized BGK equation with 3-dimensional velocity space.

\subsection{The Equation}

Consider the following stationary equation in a half space

$$
\begin{aligned}
(v+u) \partial_{x} \varphi+\varphi-<\varphi M^{\frac{1}{2}}>M^{\frac{1}{2}} & =0 \\
\varphi(0, v) & =k(v), \quad v+u>0
\end{aligned}
$$

with $x \in[0, \infty), v \in \mathbf{R}, u \in \mathbf{R}$. Here we denoted by $M$ the centered, reduced Maxwellian $M=(2 \pi)^{-\frac{1}{2}} \exp \left(-\frac{v^{2}}{2}\right)$ and we define $\left\langle f>:=\int_{\mathbf{R}} f(v) d v\right.$, if $f$ is integrable. The following proposition recalls the essentials of the existence, uniqueness and asymptotic behavior results already known for this simple model, see Kaper [13] and Greenberg et al. [11].

Proposition 2.1 If $u \geq 0$ then the above problem has $\forall k \in \mathcal{L}^{2}((1+|v|) d v)$ a unique solution $\varphi \in \mathcal{L}^{\infty}\left(d x, \mathcal{L}^{2}((1+|v|) d v)\right)$. If $u<0$ there exists again a unique solution once the flux $\int(v+u) \varphi(x, v) M^{\frac{1}{2}} d v$, which is independent of $x$, is assigned an arbitrary value $m \in \mathbf{R}$.

Moreover $\varphi(x, v) \rightarrow \lambda_{\infty} M^{\frac{1}{2}}$ as $x \rightarrow \infty$, where $\lambda_{\infty} \in \mathbf{R}$.

As explained in the introduction, for the purpose of domain decompositions we are interested only in the asymptotic state $\lambda_{\infty}$ and in the reflected density $\varphi(0, v), v+u<0$. We shall call "albedo operator" the linear operator $\varphi(0, v) \mapsto \varphi(0,-v-2 u), v+u>0$. We will now describe a numerical procedure to compute these values.

\subsection{The Numerical Method for $u>0$}

The main idea behind the method is to solve the gas dynamics equations associated to the model Boltzmann equation and then to use the Chapman-Enskog expansion as an approximate solution. 


\section{Computation of the asymptotic states:}

Instead of

$$
\begin{aligned}
(v+u) \partial_{x} \varphi+\varphi-<\varphi M^{\frac{1}{2}}>M^{\frac{1}{2}} & =0, u>0 \\
\varphi(0, v) & =k(v), \quad v+u>0
\end{aligned}
$$

consider the adjoint equation

$$
\begin{aligned}
-(v+u) \partial_{x} \psi+\psi-<\psi M^{\frac{1}{2}}>M^{\frac{1}{2}} & =0, u>0 \\
\psi(0, v) & =0, \quad v+u<0 .
\end{aligned}
$$

Here, according to the proposition above, an additional flux condition is needed. We put

$$
<(v+u) M^{\frac{1}{2}} \psi>=1
$$

The usual form of the above equation is seen by transforming $v \rightarrow-v, u \rightarrow-u$, which gives

$$
\begin{aligned}
(v+u) \partial_{x} \psi+\psi-<\psi M^{\frac{1}{2}}>M^{\frac{1}{2}} & =0 \quad u<0 \\
\psi(0, v) & =0, \quad v+u>0 \\
<(v+u) \psi M^{\frac{1}{2}}> & =-1 .
\end{aligned}
$$

This equation will now be solved approximately. We proceed as in the Chapman-Enskog expansion method. Integrating the equation over the velocity space gives

$$
\partial_{x}<(v+u) \psi M^{\frac{1}{2}}>=\partial_{x}<v \psi M^{\frac{1}{2}}>+u \partial_{x}<\psi M^{\frac{1}{2}}>=0 .
$$

The macroscopic moment is

$$
\tilde{\Theta}_{1}:=<\psi M^{\frac{1}{2}}>
$$

The conservation equation is then

$$
\partial_{x}<v \psi M^{\frac{1}{2}}>+u \partial_{x} \tilde{\Theta}_{1}=0 .
$$

Defining $L \psi:=\psi-<\psi M^{\frac{1}{2}}>M^{\frac{1}{2}}$, one gets, with $v M^{\frac{1}{2}}=L\left(v M^{\frac{1}{2}}\right)$ and (2.3):

$$
<v M^{\frac{1}{2}} \psi>=<L\left(v M^{\frac{1}{2}}\right) \psi>=<v M^{\frac{1}{2}} L \psi>=-\partial_{x}<v M^{\frac{1}{2}}(v+u) \psi>.
$$

Here we substitute the zeroth order approximation for $\psi, \psi \sim \tilde{\Theta}_{1} M^{\frac{1}{2}}$ :

$$
<v M^{\frac{1}{2}} \psi>=-\partial_{x}\left(<v^{2} M>\tilde{\Theta}_{1}\right)-u \partial_{x}<v M>\tilde{\Theta}_{1}=-\partial_{x} \tilde{\Theta}_{1} .
$$

This results in

$$
u \partial_{x} \Theta_{1}-\partial_{x}^{2} \Theta_{1}=0, \quad u<0
$$


as an approximate equation for $\tilde{\Theta}_{1}=<\psi M^{\frac{1}{2}}>$. This is the analogue of the gas dynamics equation for the model considered here. Its solution can be determined exactly up to 2 parameters:

$$
\Theta_{1}(x)=A e^{u x}+\Theta_{\infty}^{(1)}, \quad \text { where } \quad A, \Theta_{\infty}^{(1)} \in \mathbf{R}, u<0 .
$$

Next we compute the first approximation $\psi_{1}$ of $\psi$ by the following equation

$$
\begin{aligned}
(v+u) \partial_{x} \psi_{1}+\psi_{1}-\Theta_{1} M^{\frac{1}{2}} & =0, u<0 \\
\psi_{1}(0, v) & =0 \quad v+u>0
\end{aligned}
$$

where the solution $\Theta_{1}$ of the model gas dynamic equation is substituted to $\left\langle\psi M^{\frac{1}{2}}\right\rangle$ in (2.3). Notice that $<(v+u) \psi_{1} M^{\frac{1}{2}}>$ is no longer independent of $x$.

However, the solution of (2.4) can be given explicitely:

$$
\psi_{1}(x, v)=\left\{\begin{array}{l}
\Theta_{\infty}^{(1)}\left(1-e^{-\frac{x}{v+u}}\right)+X A\left(e^{u x}-e^{-\frac{x}{v+u}}\right), v+u<0 \\
\Theta_{\infty}^{(1)}+A e^{u x} X, \quad v+u>0
\end{array}\right.
$$

where $X:=\frac{1}{1+u(v+u)}$. We determine $\Theta_{\infty}^{(1)}$ and $A$ by

$$
<(v+u)\left\{\begin{array}{c}
\psi_{1}(\infty, v) \\
\psi_{1}(0, v)
\end{array}\right\} M^{\frac{1}{2}}>=-1
$$

the closest analogue to $\left\langle(v+u) M^{\frac{1}{2}} \psi>=-1\right.$. The $\psi$ approximation can be iterated. Consider the equation for the remaining term $\psi-\psi_{1}$ : With (2.3) and (2.4) it is

$$
\begin{gathered}
(v+u) \partial_{x}\left(\psi-\psi_{1}\right)+\left(\psi-\psi_{1}\right)-\left(<\left(\psi-\psi_{1}\right) M^{\frac{1}{2}}>+<\psi_{1} M^{\frac{1}{2}}>-\Theta_{1}\right) M^{\frac{1}{2}}=0, u<0 \\
\left(\psi-\psi_{1}\right)(0, v)=0 \quad v+u>0
\end{gathered}
$$

Defining the first order approximation of the macroscopic moment $\tilde{\Theta}_{2}:=<\psi M^{\frac{1}{2}}>-\Theta_{1}$ one can derive in the same way as above an approximate equation for $\tilde{\Theta}_{2}$, namely

$$
u \partial_{x} \Theta_{2}-\partial_{x}^{2} \Theta_{2}=<\psi_{1} M^{\frac{1}{2}}>-\Theta_{1}
$$

$\Theta_{2}$ is uniquely determined up to 2 Parameters $\Theta_{\infty}^{(2)}, B$. The approximation $\psi_{2}$ of $\psi-\psi_{1}$ solves

$$
\begin{gathered}
(v+u) \partial_{x} \psi_{2}+\psi_{2}-\left(\Theta_{2}+<\psi_{1} M^{\frac{1}{2}}>-\Theta_{1}\right) M^{\frac{1}{2}}=0 \\
\psi_{2}(0, v)=0, \quad v+u>0 .
\end{gathered}
$$

Here again $\Theta_{2}$ was used in place of $<\left(\psi-\psi_{1}\right) M^{\frac{1}{2}}>$. The 2 parameters $\Theta_{\infty}^{(2)}$ and $B$ are then determined by

$$
<(v+u)\left\{\begin{array}{l}
\psi_{2}(0, v) \\
\psi_{2}(\infty, v)
\end{array}\right\} M^{\frac{1}{2}}>=0 .
$$

The next steps of this procedure can be carried through in the same way and produce an expansion

$$
\psi \sim \psi_{1}+\psi_{2}+\ldots+\psi_{n}
$$


with

$$
\begin{gathered}
(v+u) \partial_{x} \psi_{k}+\psi_{k}-\left(\Theta_{k}+g_{k}\right) M^{\frac{1}{2}}=0, k=2 \ldots n \\
\psi_{k}(0, v)=0, v+u>0 \\
<(v+u)\left\{\begin{array}{l}
\psi_{k}(0, v) \\
\psi_{k}(\infty, v)
\end{array}\right\} M^{\frac{1}{2}}>=0
\end{gathered}
$$

where

$$
g_{k}=<\psi_{k-1} M^{\frac{1}{2}}>-\Theta_{k-1}
$$

and

$$
u \partial_{x} \Theta_{k}-\partial_{x}^{2} \Theta_{k}=g_{k}
$$

Assuming that the series

$$
\psi_{1}+\psi_{2}+\psi_{3}+\ldots
$$

converges, one can see that it is equal to the desired solution $\psi$ of equation (2.3) by the following simple calculation:

Using the above equations and equation (2.4) we obtain

$$
(v+u) \partial_{x}\left(\sum_{k=1}^{\infty} \psi_{k}\right)+\left(\sum_{k=1}^{\infty} \psi_{k}\right)-\left(\Theta_{1}+\sum_{k=2}^{\infty}\left(\Theta_{k}+g_{k}\right)\right) M^{\frac{1}{2}}=0
$$

This gives

$$
(v+u) \partial_{x}\left(\sum_{k=1}^{\infty} \psi_{k}\right)+\left(\sum_{k=1}^{\infty} \psi_{k}\right)-<\left(\sum_{k=1}^{\infty} \psi_{k}\right) M^{\frac{1}{2}}>M^{\frac{1}{2}}=0 .
$$

Moreover $\sum_{k=1}^{\infty} \psi_{k}$ satisfies the boundary condition at $x=0$ as well as the constraint required of the solution of $(2.3)$. This means that $\sum_{k=1}^{\infty} \psi_{k}$ is equal to $\psi$.

One only has to transform $v$ and $u$ backwards, $v \rightarrow-v, u \rightarrow-u$ to get the desired approximation of $(2.2), \psi(x, v)$.

The following observation is crucial for the whole scheme: If $\varphi$ is a solution of (2.1) and $\psi$ one of $(2.2)$

$$
\begin{aligned}
& \partial_{x}(<(v+u) \varphi(x, v) \psi(x, v)>) \\
& =<(v+u)\left(\partial_{x} \varphi\right)(x, v) \psi(x, v)>+<(v+u)\left(\partial_{x} \psi\right)(x, v) \varphi(x, v)> \\
& =<L(\varphi) \psi>-<L(\psi) \varphi> \\
& =<L(\psi) \varphi>-<L(\psi) \varphi> \\
& =0
\end{aligned}
$$

In other words, $\langle(v+u) \varphi \psi\rangle$ is an invariant in $x$. Using this invariant we get

$$
<(v+u) \varphi(\infty, v) \psi(\infty, v)>=<(v+u) \varphi(0, v) \psi(0, v)>
$$


and substituting gives

$$
<(v+u) \lambda_{\infty} M^{\frac{1}{2}} \psi(\infty, v)>=\int_{v+u>0}(v+u) k(v) \psi(0, v) d v .
$$

Or with $<(v+u) M^{\frac{1}{2}} \psi(x, v)>=1$

$$
\lambda_{\infty}=\int_{v+u>0}(v+u) k(v) \psi(0, v) d v
$$

where

$$
\psi(0, v) \sim \psi_{1}(0, v)+\psi_{2}(0, v)+\ldots+\psi_{n}(0, v)
$$

E. g. the first approximation $\psi_{1}(0, v)$ is explicitly

$$
\psi_{1}(0, v)=\left\{\begin{array}{ll}
\frac{1}{u}+A X, & v+u>0 \\
0, & v+u<0
\end{array} .\right.
$$

with $X$ and $A$ defined above.

Remark: The second iteration gives already such a good approximation that usually there is no need to iterate further.

\section{Computation of the Albedo Operator:}

Here we are interested in computing the outgoing density $\varphi(0, v), v+u<0$ of (2.1).

We proceed in the same way as before, except that now $\varphi(\infty, v)=\lambda_{\infty} M^{\frac{1}{2}}$ is known. Therefore (2.1) can be used directly.

Here again, the gas dynamics equation is $u \partial_{x} \Theta_{1}-\partial_{x}^{2} \Theta_{1}=0$ but with $u>0$. Demanding that the solution be finite at infinity, one has

$$
\Theta_{1}=A e^{u x}+\Theta_{\infty}^{(1)}=\Theta_{\infty}^{(1)}
$$

Substituting as before $\Theta_{1}$ into (2.1) gives

$$
\begin{aligned}
(v+u) \partial_{x} \varphi_{1}+\varphi_{1}-\Theta_{\infty}^{(1)} M^{\frac{1}{2}} & =0 \\
\varphi_{1}(0, v) & =k(v), v+u>0
\end{aligned}
$$

The solution is

$$
\varphi_{1}(x, v)=\left\{\begin{array}{ll}
e^{-\frac{x}{v+u}} k(v)+\Theta_{\infty}^{(1)}\left(1-e^{-\frac{x}{v+u}}\right) M^{\frac{1}{2}}, & v+u>0 \\
\Theta_{\infty}^{(1)} M^{\frac{1}{2}}, & v+u<0
\end{array} .\right.
$$

$\varphi(\infty, v)=\lambda_{\infty} M^{\frac{1}{2}}$ gives $\Theta_{\infty}^{(1)}=\lambda_{\infty}$ and therefore $\varphi_{1}(0, v)=\lambda_{\infty} M^{\frac{1}{2}}, v+u<0$. Here, the next iteration step is needed to get the first nontrivial approximation of the albedo operator. Considering the equation for $\varphi-\varphi_{1}$ gives the second-step gas dynamics equation:

$$
u \partial_{x} \Theta_{2}-\partial_{x}^{2} \Theta_{2}=<\varphi_{1} M^{\frac{1}{2}}>-\lambda_{\infty} .
$$


One solves as before

$$
\begin{aligned}
(v+u) \partial_{x} \varphi_{2}+\varphi_{2}-\left(\Theta_{2}+<\varphi_{1} M^{\frac{1}{2}}>-\lambda_{\infty}\right) M^{\frac{1}{2}} & =0 \\
\varphi_{2}(0, v) & =0, v+u>0,
\end{aligned}
$$

where $\Theta_{2}$ is substituted into the equation for $\varphi-\varphi_{1}$. This yields $\varphi_{2}$. Iterating this we end up with an approximation of

$$
\varphi \sim \varphi_{1}+\ldots+\varphi_{n}
$$

and in particular

$$
\varphi(0, v) \sim \varphi_{1}(0, v)+\ldots+\varphi_{n}(0, v), v+u<0 .
$$

\section{Remark (The Maxwell conditions):}

The following method was developed by Maxwell [17] to derive approximate boundary conditions: In order to determine $\lambda_{\infty}$, one equalizes the half-fluxes at the boundary and at infinity, i.e.

$$
\int_{v+u>0}(v+u) \varphi(0, v) M^{\frac{1}{2}} d v=\int_{v+u>0}(v+u) \varphi(\infty, v) M^{\frac{1}{2}} d v
$$

which means

$$
\lambda_{\infty} \int_{v+u>0}(v+u) M d v=\int_{v+u>0}(v+u) k(v) M^{\frac{1}{2}} d v
$$

or

$$
\lambda_{\infty}=\frac{\int_{v+u>0}(v+u) M^{\frac{1}{2}} k(v) d v}{\int_{v+u>0}(v+u) M d v}, \quad \forall u \geq 0 .
$$

Of course, this equality is in general wrong on the mathematical level but can provide correct orders of magnitudes. In many applications this method or simply the matching by equality of moments (i.e. of local macroscopic quantities) are chiefly used to define the coupling conditions at the interface. They amount essentially to assume that there is no transition layer between the kinetic region and the gas dynamics region.

Our numerical results show that it seems to be valid for high Mach numbers, whereas for small or moderate Mach numbers the results differ from those given by the above method. The Maxwell approximation of the albedo operator is simply

$$
\varphi(0, v)=\varphi(\infty, v)=\lambda_{\infty} M^{\frac{1}{2}}, v+u<0 .
$$

\subsection{Numerical Method for $u=0$}

For $u=0$, there is some degeneracy in the equation and the method must be changed slightly. We describe the procedure and show its equivalence to the variational methods for $u=0$. 


\section{Computation of the asymptotic states:}

The equation is

$$
\begin{aligned}
v \partial_{x} \varphi+\varphi-<\varphi M^{\frac{1}{2}}>M^{\frac{1}{2}} & =0 \\
\varphi(0, v) & =k(v), v>0
\end{aligned}
$$

Here no additional condition on $\varphi$ is needed, but if $\varphi$ is the (unique) bounded (in $x$ ) solution, then

$$
\int v \varphi(x, v) M^{\frac{1}{2}} d v=0 .
$$

Consider again the adjoint equation

$$
\begin{aligned}
-v \partial_{x} \psi+\psi-<\psi M^{\frac{1}{2}}>M^{\frac{1}{2}} & =0 \\
\psi(0, v) & =0 \quad v<0
\end{aligned}
$$

and choose the constraint $\left\langle v \psi M^{\frac{1}{2}}\right\rangle=1$. Transforming $v \rightarrow-v$ gives

$$
\begin{aligned}
v \partial_{x} \psi+\psi-<\psi M^{\frac{1}{2}}>M^{\frac{1}{2}} & =0 \\
\psi(0, v) & =0, \quad v>0 \\
<v \psi M^{\frac{1}{2}}> & =-1
\end{aligned}
$$

According to the above remark, the solution of (2.7) is necessarily unbounded. In fact, $\psi$ grows linearly in $x$. Taking this into account, we define

$$
\chi:=\psi-x M^{\frac{1}{2}}+v M^{\frac{1}{2}}, \quad \text { i.e. } \quad \psi=\chi+(x-v) M^{\frac{1}{2}},
$$

which gives

$$
\begin{aligned}
v \partial_{x} \chi+\chi-<\chi M^{\frac{1}{2}}>M^{\frac{1}{2}} & =0 \\
\chi(0, v) & =v M^{\frac{1}{2}}, v>0 \\
<v \chi M^{\frac{1}{2}}> & =0 .
\end{aligned}
$$

According to the proposition above, there exists a unique bounded solution $\chi$ of this equation. $\psi$ is given by

$$
\psi=\chi+(x-v) M^{\frac{1}{2}} .
$$

The iterative procedure for (2.8) now parallels to the case $u>0$ : The model gas dynamics equation is simply $\partial_{x}^{2} \Theta_{1}=0$ with the solution $\Theta_{1}=\Theta_{\infty}^{(1)}$ (using the boundedness at infinity). Substituting $\Theta_{1}$ for $<\chi M^{\frac{1}{2}}>$ in (2.8) gives

$$
\chi_{1}(x, v)=\left\{\begin{array}{ll}
e^{-\frac{x}{v}} v M^{\frac{1}{2}}+\Theta_{\infty}^{(1)}\left(1-e^{-\frac{x}{v}}\right) M^{\frac{1}{2}}, & v>0 \\
\Theta_{\infty}^{(1)} M^{\frac{1}{2}}, & v<0
\end{array} .\right.
$$


The condition $<v M^{\frac{1}{2}} \chi_{1}(\infty, v)>=0$ being automatically fulfilled, $\left\langle v \chi_{1}(0, v) M^{\frac{1}{2}}>=0\right.$ gives

$$
\Theta_{\infty}^{(1)}=\frac{\int_{v>0} v^{2} M d v}{\int_{v>0} v M d v}=\frac{\sqrt{2 \pi}}{2} .
$$

Further iteration yields $\chi \sim \chi_{1}+\chi_{2}+\ldots+\chi_{n}$ where, for $k=2 \ldots n, \chi_{k}$ solves

$$
\begin{aligned}
v \partial_{x} \chi_{k}+\chi_{k}-\left(\Theta_{k}+g_{k}\right) M^{\frac{1}{2}} & =0 \\
\chi_{k}(0, v) & =0, v>0 \\
<v\left\{\begin{array}{l}
\chi_{k}(0, v) \\
\chi_{k}(\infty, v)
\end{array}\right\} M^{\frac{1}{2}}> & =0
\end{aligned}
$$

with

$$
g_{k}=<\chi_{k-1} M^{\frac{1}{2}}>-\Theta_{k-1}
$$

and

$$
\partial_{x}^{2} \Theta_{k}=g_{k}
$$

Taking $\psi=\chi+(x-v) M^{\frac{1}{2}}$ and transforming $v \rightarrow-v$ we get $\psi \sim \psi_{1}+\ldots+\psi_{n}$ as approximate solution of (2.6). As before, $\lambda_{\infty}$ is computed by

$$
\lambda_{\infty}=\int_{v>0} v k(v) \psi(0, v) d v
$$

with $\psi(0, v)=\psi_{1}(0, v)+\psi_{2}(0, v)+\ldots+\psi_{n}(0, v)$.

E. g. the first approximation for $\psi(0, v)$ is

$$
\psi_{1}(0, v)=\left\{\begin{array}{ll}
0, & v<0 \\
\left(\Theta_{\infty}^{(1)}+v\right) M^{\frac{1}{2}}, & v>0
\end{array} .\right.
$$

The albedo operator is computed as in 2.2 .

We remark that $\lambda_{\infty}$ computed in this section is exactly the limit of $\lambda_{\infty}$ computed in section 2.2 as u tends to 0 .

\section{Equivalence to the variational approach:}

If $u=0$ the first step of our method is equivalent to an approach developed by Cercignani [4], Golse [10] and Loyalka-Ferziger [15] to compute the slip coefficients in the boundary conditions for gas dynamic equations. The variational method is based on the observation that for $u=0$, in addition to $\left\langle v M^{\frac{1}{2}} \varphi(x, v)>\right.$ the quantity $\left\langle v L^{-1}\left(M^{\frac{1}{2}} v\right) \varphi(x, v)>\right.$ also is an invariant (with respect to $x$ ) of the half-space equation, where $L$ is defined as $L \psi:=\psi-\left\langle\psi M^{\frac{1}{2}}>M^{\frac{1}{2}}\right.$. This is particular to the case $u=0$. We recall the result of this variational procedure shortly and refer the reader to the literature cited above for more details: 
$\varphi(0, v)$ is assumed to be equal to $C M^{\frac{1}{2}}$ for $v<0, C$ a constant s.t. $\int v \varphi(0, v) M^{\frac{1}{2}} d v=0$, i.e.

$$
C=\frac{\int_{v>0} v k(v) M^{\frac{1}{2}} d v}{\int_{v>0} v M d v} .
$$

Then with $L^{-1}\left(v M^{\frac{1}{2}}\right)=v M^{\frac{1}{2}}$ one computes

$$
\begin{aligned}
<v^{2} \varphi(0, v) M^{\frac{1}{2}}> & =<v L^{-1}\left(v M^{\frac{1}{2}}\right) \varphi(0, v)> \\
= & <v L^{-1}\left(v M^{\frac{1}{2}}\right) \varphi(\infty, v)>=\left\langle v^{2} M^{\frac{1}{2}} \varphi(\infty, v)>\right.
\end{aligned}
$$

Or in other words

$$
\begin{aligned}
& \int_{v>0} v^{2} k(v) M^{\frac{1}{2}} d v+\int_{v<0} v^{2} C M d v=<v^{2} M>\lambda_{\infty}=\lambda_{\infty} \\
& \lambda_{\infty}=\int_{v>0} v^{2} k(v) M^{\frac{1}{2}} d v+\int_{v>0} v k(v) M^{\frac{1}{2}} d v \frac{\int_{v<0} v^{2} M d v}{\int_{v>0} v M d v}
\end{aligned}
$$

To see that our method yields the same equation we only have to remember the definition of $\Theta_{\infty}^{(1)}$ and $\psi_{1}$. Then

$$
\begin{aligned}
\lambda_{\infty} & =\int_{v>0} v k(v)\left(v+\Theta_{\infty}^{(1)}\right) M^{\frac{1}{2}} d v \\
& =\int_{v>0} v k(v) \psi_{1}(0, v) d v .
\end{aligned}
$$

This is exactly the asymptotic value obtained by the first iteration step of our iterative method for $u=0$.

Equivalently, our approach uses an extension to general $u$ of the well-known invariant $L^{-1}\left(v M^{\frac{1}{2}}\right)$ in the case $u=0$.

\subsection{Results}

We used $k(v)=v M^{\frac{1}{2}}$ to get for $u=0$ the usual velocity slip coefficient, see Cercignani [6]. The asymptotic values for $u=0$ are computed as 1.2533 for the Maxwell method described in the remark at the end of section 2.2, 1.4245 for the first step or the variational method and 1.4348 for the second step.

We compare these values with the calculations of Sone/Onishi [20], who found for the velocity slip coefficient the value 1.01619 which has to be multiplied by $\sqrt{2}$ to get 1.4371 . The same result can be found also in Cercignani [6], where the constant for the slip 
coefficient has to be multiplied by $\sqrt{\frac{\pi}{2}}$.

The results for $u>0$ are shown for the Maxwell method and the first two steps of the method in Figure 1.

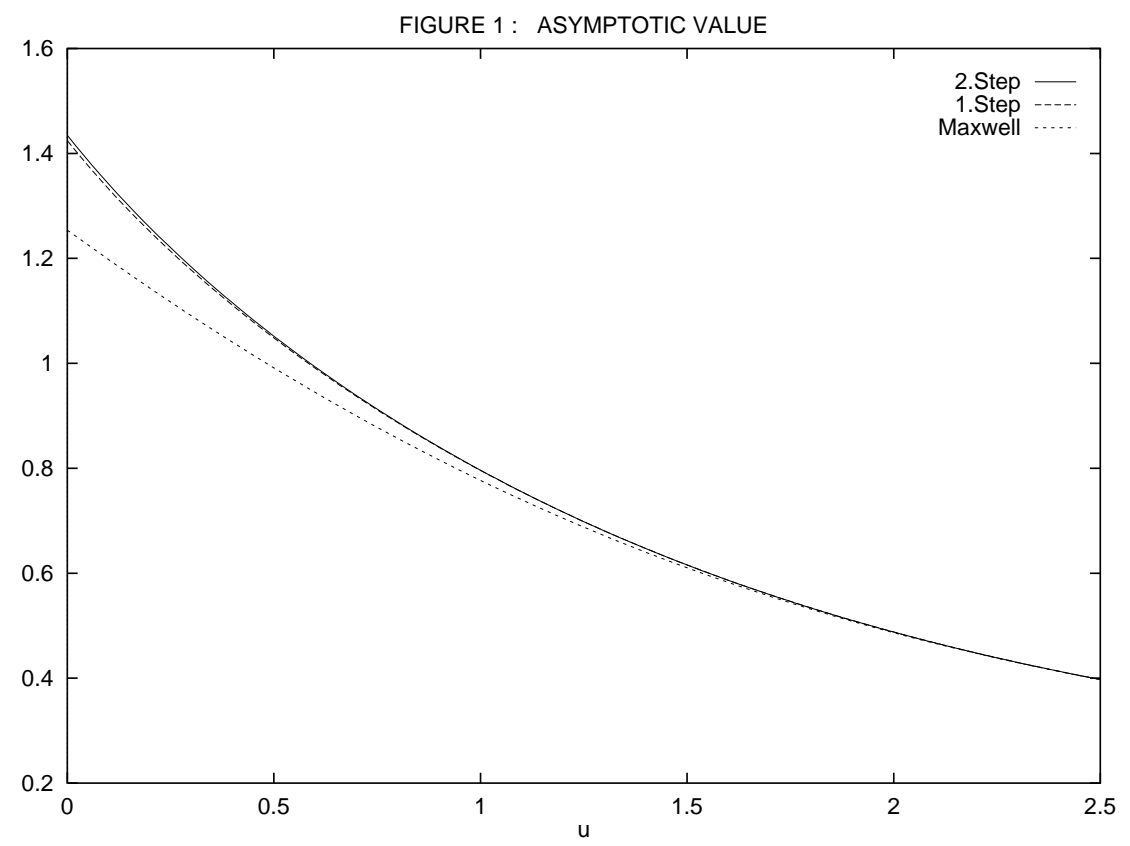

The computation of the albedo multiplied by $M^{-\frac{1}{2}}$ in the case $u=0$ is shown in Figure 2 where we computed the true outgoing distribution by numerical integration of a formula to be found e.g. in Cercignani [4]. Figure 3 shows the albedo for $u=1$ multiplied by $M^{-\frac{1}{2}}$. We calculated the 'true' solution for $u=1$ by a direct computation using a standard iteration scheme. 


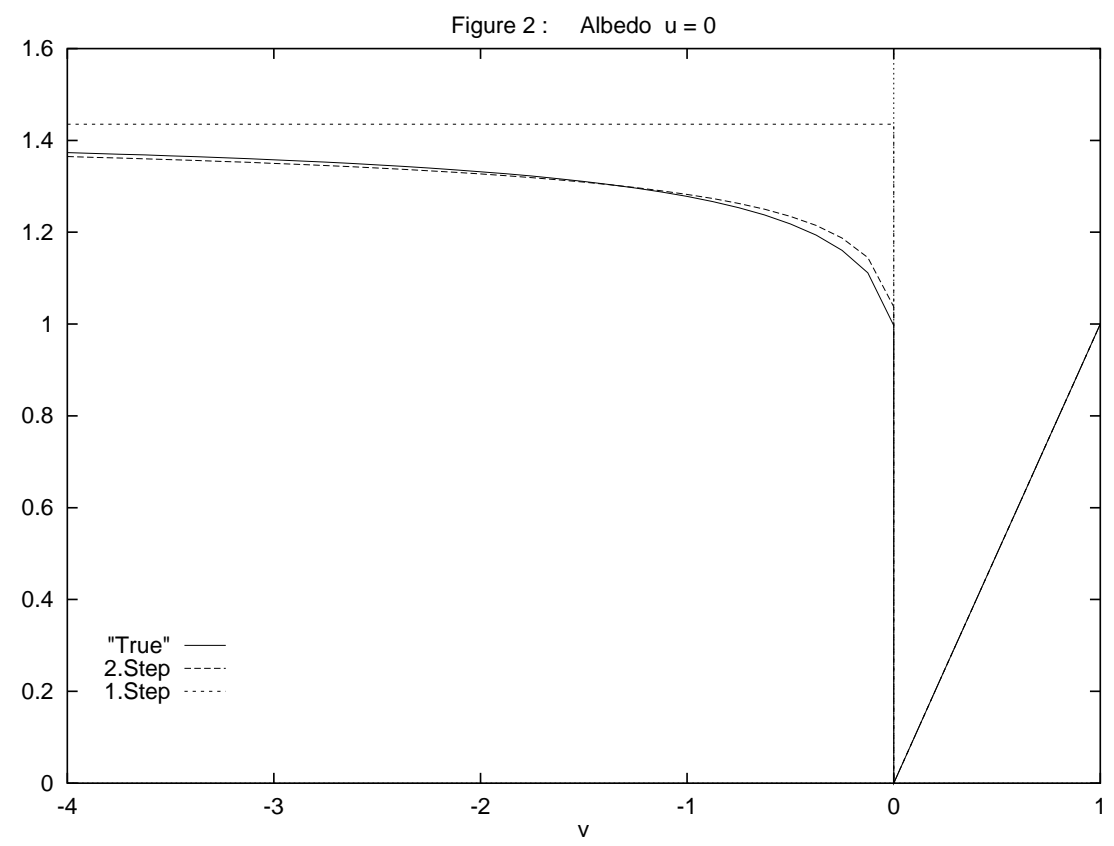

Figure 3: $\quad$ Albedo $\mathrm{u}=1$

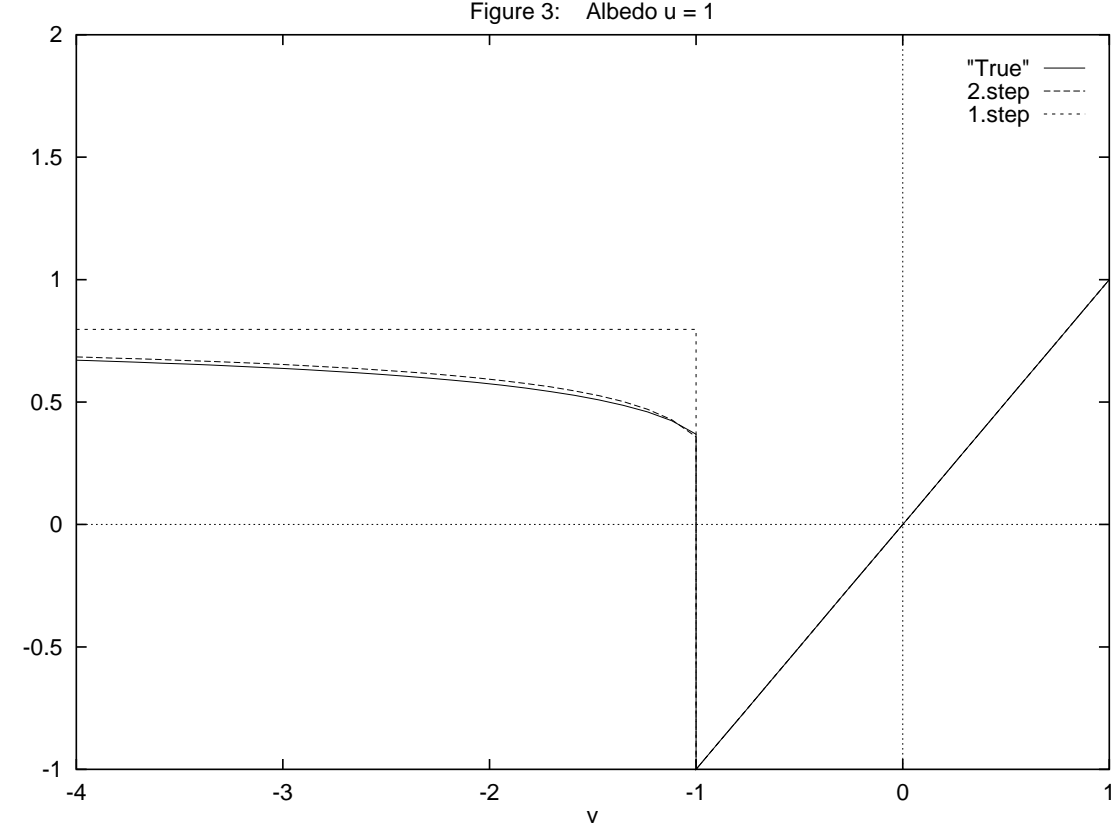

For a comparison of these results see Coron [7], where the asymptotic values were computed by a spectral method. 


\section{The 3-Dimensional BGK Equation}

Here the linearized form of the BGK equation will be considered, i.e. the usual collision operator of the linearized Boltzmann equation is replaced by a projection operator describing the relaxation to a Maxwellian.

\subsection{The Equation}

Consider

$$
\begin{aligned}
& \left(v_{1}+u\right) \partial_{x} \varphi+\varphi-\Pi^{B G K} \varphi=0 \\
& \varphi(0, v)=k(v), v_{1}+u>0,
\end{aligned}
$$

where

$$
\Pi^{B G K} \varphi:=M^{\frac{1}{2}}\left(<\varphi M^{\frac{1}{2}}>+\sum_{i=1}^{3}<\varphi v_{i} M^{\frac{1}{2}}>v_{i}+<\varphi \frac{|v|^{2}-3}{3} M^{\frac{1}{2}}>\frac{|v|^{2}-3}{2}\right),
$$

$x \in[0, \infty], v=\left(v_{1}, v_{2}, v_{3}\right) \in \mathbf{R}^{3}, u \in \mathbf{R},<f>:=\int_{\mathbf{R}^{3}} f(v) d v, M=(2 \pi)^{-\frac{3}{2}} \exp \left(-\frac{|v|^{2}}{2}\right)$.

The existence and uniqueness theory as well as the asymptotic behavior are summarized in the following proposition, see Arthur and Cercignani [2], Siewert and Thomas [19] or Greenberg et al. [11].

Proposition 3.1 If $u>c=\sqrt{\frac{5}{3}}$ then the above problem has $\forall k \in \mathcal{L}^{2}((1+|v|) d v)$ a unique solution $\varphi \in \mathcal{L}^{\infty}\left(d x, \mathcal{L}^{2}((1+|v|) d v)\right)$.

If $0 \leq u<c$ it has a unique solution if $\int v_{1} \varphi(\infty, v) M^{\frac{1}{2}} d v$ is fixed to 0 .

4 conditions are to be fixed, if $-c<u<0.5$ conditions are needed, if $u<-c$.

The treatment can be simplified by splitting the BGK-equation into two parts, the shear flow part, which is after some manipulations equivalent to the equation treated in section 2 and the heat transfer part (see Cercignani [6]). The latter is governed by

$$
\begin{aligned}
\left(v_{1}+u\right) \partial_{x} \varphi+\varphi-\Pi \varphi & =0 \\
\varphi(0, v) & =k(v), \quad v_{1}+u>0
\end{aligned}
$$

where

$$
\Pi \varphi:=M^{\frac{1}{2}}\left(<\varphi M^{\frac{1}{2}}>+<v_{1} \varphi M^{\frac{1}{2}}>v_{1}+<\varphi \frac{|v|^{2}-3}{3} M^{\frac{1}{2}}>\frac{|v|^{2}-3}{2}\right) .
$$

If $u>c$ we need no additional condition. For $0 \leq u<c$ one condition is needed. For $-c<u<0$ two conditions and for $u<-c$ three conditions are necessary. 
The asymptotic state is

$$
\varphi(\infty, v)=\left(a_{\infty}+b_{\infty} v_{1}+c_{\infty} \frac{|v|^{2}-3}{2}\right) M^{\frac{1}{2}} .
$$

The numerical scheme we developed in Section 2 will now be extended to this case to compute $a_{\infty}, b_{\infty}, c_{\infty}$.

\subsection{Numerical Method for $u>0$}

We consider (3.1) with the condition

$$
<v_{1} \varphi(\infty, v) M^{\frac{1}{2}}>=0,
$$

if $0<u<c$ and without any condition if $u>c$. The adjoint equation is

$$
\begin{aligned}
-\left(v_{1}+u\right) \partial_{x} \psi+\psi-\Pi \psi & =0, & u>0 \\
\psi(0, v) & =0, & v_{1}+u<0
\end{aligned}
$$

with 2 conditions if $0<u<c$, respectively all 3 conditions if $u>c$ out of the full set of relations

$$
<\left(v_{1}+u\right) \psi M^{\frac{1}{2}}\left(\begin{array}{c}
1 \\
v_{1} \\
|v|^{2}
\end{array}\right)>=\left(\begin{array}{c}
1 \\
1 \\
1
\end{array}\right) .
$$

We transform again $v \rightarrow-v, u \rightarrow-u$ and consider

$$
\begin{aligned}
\left(v_{1}+u\right) \partial_{x} \psi+\psi-\Pi \psi & =0, & u<0 \\
\psi(0, v) & =0, & v_{1}+u>0
\end{aligned}
$$

with 2 resp. 3 conditions out of the full set of relations

$$
<\left(v_{1}+u\right)\left(\begin{array}{c}
1 \\
v_{1} \\
|v|^{2}
\end{array}\right) \psi M^{\frac{1}{2}}>=\left(\begin{array}{c}
-1 \\
-1 \\
-1
\end{array}\right) .
$$

By the usual Chapman Enskog procedure one can see that the macroscopic moments

$$
\tilde{\rho}_{1}:=<\psi M^{\frac{1}{2}}>, \tilde{u}_{1}:=<v_{1} \psi M^{\frac{1}{2}}>, \tilde{\Theta}_{1}:=<\frac{|v|^{2}-3}{3} \psi M^{\frac{1}{2}}>
$$

approximately solve the stationary linearized Navier-Stokes equations associated to the BGK collision kernel:

$$
\begin{aligned}
\partial_{x}\left(u_{1}+u \rho_{1}\right) & =0 \\
\partial_{x}\left(\Theta_{1}+\rho_{1}+u u_{1}-\partial_{x} \frac{4}{3} u_{1}\right) & =0 \\
\partial_{x}\left(3 u \Theta_{1}-2 u \rho_{1}-5 \partial_{x} \Theta_{1}\right) & =0
\end{aligned}
$$


These can be solved exactly up to 5 free parameters $\rho_{\infty}^{(1)}, u_{\infty}^{(1)}, \Theta_{\infty}^{(1)}, A, B$ :

$$
\begin{aligned}
\rho_{1}(x) & =\rho_{\infty}^{(1)}+A \gamma_{0} e^{\lambda_{1} x}+B \gamma_{0} e^{\lambda_{2} x} \\
u_{1}(x) & =-u_{\infty}^{(1)}+A e^{\lambda_{1} x}+B e^{\lambda_{2} x} \\
\Theta_{1}(x) & =\Theta_{\infty}^{(1)}+A \gamma_{1} e^{\lambda_{1} x}+B \gamma_{2} e^{\lambda_{2} x}
\end{aligned}
$$

where

$$
\begin{aligned}
& \gamma_{0}=-\frac{1}{u}, \gamma_{i}=\frac{4}{3} \lambda_{i}+\left(\frac{1}{u}-u\right), i=1,2 \\
& \lambda_{i}=\frac{1}{40}\left(27 u-\frac{15}{u}+(-1)^{i}\left(9 u^{2}+\left(\frac{15}{u}\right)^{2}+390\right)^{1 / 2}\right), i=1,2
\end{aligned}
$$

with $\lambda_{1}<0$, if $u<-c$ and $\lambda_{1}>0$,if $-c<u<0$ and $\lambda_{2}<0$, forall $u<0$. We define

$$
\begin{aligned}
S_{\infty}^{(1)} & =\left(\rho_{\infty}^{(1)}-u_{\infty}^{(1)} v_{1}+\Theta_{\infty}^{(1)} \frac{|v|^{2}-3}{2}\right) M^{\frac{1}{2}} \\
T_{i} & =\left(\gamma_{0}+v_{1}+\gamma_{i} \frac{|v|^{2}-3}{2}\right) M^{\frac{1}{2}} \\
X_{i} & =\left(1+\lambda_{i}\left(v_{1}+u\right)\right)^{-1}, i=1,2 .
\end{aligned}
$$

The first approximation $\psi_{1}$ for $\psi$ can now be calculated from

$$
\begin{aligned}
& \left(v_{1}+u\right) \partial_{x} \psi_{1}+\psi_{1}-\left(\rho_{1}+u_{1} v+\Theta_{1} \frac{|v|^{2}-3}{2}\right) M^{\frac{1}{2}}=0 \\
& \psi_{1}(0, v)=0 \quad v_{1}+u>0
\end{aligned}
$$

as

$$
\psi_{1}(x, v)=\left\{\begin{array}{l}
e^{\lambda_{1} x} A X_{1} T_{1}+e^{\lambda_{2} x} B X_{2} T_{2}+S_{\infty}^{(1)} \\
\text { if } v_{1}+u<0 \\
\left(1-e^{-\frac{x}{v_{1}+u}}\right) S_{\infty}^{(1)}+A X_{1} T_{1}\left(e^{\lambda_{1} x}-e^{-\frac{x}{v+u}}\right)+B X_{2} T_{2}\left(e^{\lambda_{2} x}-e^{-\frac{x}{v+u}}\right) \\
\text { if } v_{1}+u>0
\end{array}\right.
$$

We determine $A, B, \rho_{\infty}^{(1)}, u_{\infty}^{(1)}, \Theta_{\infty}^{(1)}$ by the following 5 conditions if $u<-c$, and by 4 conditions out of the following 5 and the requirement $A=0$, s.t. $\rho_{1}, u_{1}, \Theta_{1}$ are finite at infinity, if $-c<u<0$ :

$$
<\left(v_{1}+u\right)\left\{\begin{array}{l}
\psi_{1}(\infty, v) \\
\psi_{1}(0, v)
\end{array}\right\}\left(\begin{array}{c}
1 \\
v_{1} \\
|v|^{2}
\end{array}\right) M^{\frac{1}{2}}>=\left(\begin{array}{c}
-1 \\
-1 \\
-1
\end{array}\right) .
$$


The further iteration steps are done in the same way as indicated in section 2.2., ending up with $\psi \sim \psi_{1}+\ldots+\psi_{n}$, where $\psi_{k}$ solves for $k=2 \ldots n$ the equations

$$
\begin{aligned}
& \left(v_{1}+u\right) \partial_{x} \psi_{k}+\psi_{k}-\left(\rho_{k}+u_{k} v_{1}+\Theta_{k} \frac{|v|^{2}-3}{2}+g_{k}^{(1)}+g_{k}^{(2)} v_{1}+g_{k}^{(3)}\left(\frac{|v|^{2}-3}{2}\right)\right) M^{\frac{1}{2}}=0 \\
& \psi_{k}(0, v)=0, v_{1}+u>0 \\
& <\left(v_{1}+u\right) M^{\frac{1}{2}}\left(\begin{array}{c}
1 \\
v_{1} \\
|v|^{2}
\end{array}\right) \psi(x, v)>=\left(\begin{array}{c}
0 \\
0 \\
0
\end{array}\right)
\end{aligned}
$$

and

$$
\begin{aligned}
& \partial_{x}\left(u_{k}+u \rho_{k}\right)=g_{k}^{(1)} \\
& \partial_{x}\left(\Theta_{k}+\rho_{k}+u u_{k}-\frac{4}{3} \partial_{x} u_{k}\right)=g_{k}^{(2)} \\
& \partial_{x}\left(-5 \partial_{x} \Theta_{k}+3 u \Theta_{k}-2 u \rho_{k}\right)=3 g_{k}^{(3)}-2 g_{k}^{(1)} \\
& g_{k}^{(1)}=<\psi_{k-1} M^{\frac{1}{2}}>-\rho_{k-1} \\
& g_{k}^{(2)}=<\psi_{k-1} v_{1} M^{\frac{1}{2}}>-u_{k-1} \\
& g_{k}^{(3)}=<\psi_{k-1} \frac{|v|^{2}-3}{3} M^{\frac{1}{2}}>-\Theta_{k-1} .
\end{aligned}
$$

By transforming $v \rightarrow-v$ and $u \rightarrow-u$ backwards we get an approximation for the solution $\psi(x, v)$ of $(3.2)$. The invariance in $x$ of

$$
<\left(v_{1}+u\right) \varphi(x, v) \psi(x, v)>
$$

established as in section 2 and that of

$$
<\left(v_{1}+u\right) M^{\frac{1}{2}}\left(\begin{array}{c}
1 \\
v_{1} \\
|v|^{2}
\end{array}\right) \varphi(x, v)>
$$

gives us the equations we need to determine

$$
\begin{gathered}
\varphi(\infty, v)=\left(a_{\infty}+c_{\infty} \frac{|v|^{2}-3}{2}\right) M^{\frac{1}{2}} \quad \text { for } 0<u<c, \\
\varphi(\infty, v)=\left(a_{\infty}+b_{\infty} v_{1}+c_{\infty} \frac{|v|^{2}-3}{2}\right) M^{\frac{1}{2}} \text { for } u>c .
\end{gathered}
$$

Remark that $b_{\infty}=0$ for $0<u<c$ since we imposed the condition $<v_{1} \varphi(\infty, v) M^{\frac{1}{2}}>=0$ on the solution $\varphi$ of (3.1). The first equation is

$$
<\left(v_{1}+u\right) \varphi(\infty, v) \psi(\infty, v)>=<\left(v_{1}+u\right) \psi(0, v) \varphi(0, v)>
$$


Moreover we use 1 equation for $0<u<c$ (respectively 2 equations for $u>c$ ) out of

$$
<\left(v_{1}+u\right)\left(\begin{array}{c}
1 \\
v_{1} \\
|v|^{2}
\end{array}\right) M^{\frac{1}{2}} \varphi(\infty, v)>=<\left(v_{1}+u\right)\left(\begin{array}{c}
1 \\
v_{1} \\
|v|^{2}
\end{array}\right) M^{\frac{1}{2}} \varphi(0, v)>.
$$

Here we substitute $\varphi(\infty, v)$ as above and $\varphi(0, v)=k(v), \quad v_{1}+u>0$.

The reflected density $\varphi(0, v), v_{1}+u<0$, that is needed in the last equations, is approximated by the Maxwell method (see the remark below). The function $\psi(0, v)$ is taken from the above approximation. For instance, the first approximation $\psi_{1}(0, v)$ for $\psi(0, v)$ is:

$$
\psi_{1}(0, v)=\left\{\begin{array}{l}
0, \quad \text { if } \quad v_{1}+u<0 \\
A X_{1} T_{1}+B X_{2} T_{2}+S_{\infty}^{(1)}, \quad \text { if } \quad v_{1}+u>0
\end{array}\right.
$$

where $X_{i}, T_{i}, i=1,2$ and $S_{\infty}^{(1)}$ are the quantities defined above after transforming $v \rightarrow$ $-v, u \rightarrow-u$. Using the constraints in equation (3.2), in particular

$$
<\left(v_{1}+u\right) \psi(\infty, v) M^{\frac{1}{2}}\left(\begin{array}{c}
1 \\
v_{1} \\
|v|^{2}
\end{array}\right)>=\left(\begin{array}{c}
1 \\
1 \\
1
\end{array}\right)
$$

provides the desired equations for $a_{\infty}, b_{\infty}, c_{\infty}$.

\section{Remark (The Maxwell Method):}

Out of the full set of relations

$$
\begin{aligned}
& \int_{v_{1}+u>0}\left(v_{1}+u\right) \varphi(\infty, v)\left(\begin{array}{c}
1 \\
v_{1} \\
|v|^{2}
\end{array}\right) M^{\frac{1}{2}} d v \\
= & \int_{v_{1}+u>0}\left(v_{1}+u\right) k(v)\left(\begin{array}{c}
1 \\
v_{1} \\
|v|^{2}
\end{array}\right) M^{\frac{1}{2}} d v
\end{aligned}
$$

we choose two conditions if $0 \leq u<c$, and all three conditions if $u>c$ to find the asymptotic states

$$
\varphi(\infty, v)=\left(a_{\infty}+c_{\infty} \frac{|v|^{2}-3}{2}\right) M^{\frac{1}{2}}
$$

and

$$
\varphi(\infty, v)=\left(a_{\infty}+b_{\infty} v_{1}+c_{\infty} \frac{|v|^{2}-3}{2}\right) M^{\frac{1}{2}} .
$$

respectively. The Maxwell approximation of the reflected density is simply

$$
\varphi(0, v)=\varphi(\infty, v), v_{1}+u<0
$$

where $\varphi(\infty, v)$ is obtained by the equations above. 


\subsection{The Numerical Method for $u=0$}

\section{Asymptotic states:}

We want to determine the asymptotic state $\varphi(\infty, v)=\left(a_{\infty}+c_{\infty} \frac{|v|^{2}-3}{2}\right) M^{\frac{1}{2}}$ of

$$
\begin{aligned}
v_{1} \partial_{x} \varphi+\varphi-\Pi \varphi & =0 \\
\varphi(0, v) & =k(v), v_{1}>0 \\
<v_{1} M^{\frac{1}{2}} \varphi(x, v)> & =0
\end{aligned}
$$

If $\varphi$ is the unique solution bounded in $x$, then $<v_{1}\left(|v|^{2}-5\right) M^{\frac{1}{2}} \varphi(x, v)>$ must be 0 or equivalently $<v_{1}|v|^{2} M^{\frac{1}{2}} \varphi(x, v)>=0$. The adjoint equation is

$$
\begin{aligned}
-v_{1} \partial_{x} \psi+\psi-\Pi \psi & =0 \\
\psi(0, v) & =0 \text { if } \quad v_{1}<0
\end{aligned}
$$

We choose the constraints

$$
<v_{1}\left(\begin{array}{c}
1 \\
|v|^{2}
\end{array}\right) \psi M^{\frac{1}{2}}>=\left(\begin{array}{c}
1 \\
15
\end{array}\right) .
$$

The transformation $v \rightarrow-v$ gives

$$
\begin{aligned}
v_{1} \partial_{x} \psi+\psi-\Pi \psi & =0 \\
\psi(0, v) & =0, \quad v_{1}>0 \\
<v_{1}\left(\begin{array}{c}
1 \\
|v|^{2}
\end{array}\right) \psi M^{\frac{1}{2}}> & =\left(\begin{array}{c}
-1 \\
-15
\end{array}\right) .
\end{aligned}
$$

As in section 2, since there is no bounded solution of (3.8), one has to look for solutions with linear growth in the variable $x$.

Defining $\chi:=\psi-x\left(|v|^{2}-5\right) M^{\frac{1}{2}}+v_{1}\left(|v|^{2}-5\right) M^{\frac{1}{2}}+v_{1} M^{\frac{1}{2}}$ gives

$$
\begin{aligned}
v_{1} \partial_{x} \chi+\chi-\Pi \chi & =0 \\
\chi(0, v) & =v_{1}\left(|v|^{2}-5\right) M^{\frac{1}{2}}+v_{1} M^{\frac{1}{2}}, v_{1}>0 \\
<v_{1}\left(\begin{array}{c}
1 \\
|v|^{2}
\end{array}\right) \chi M^{\frac{1}{2}}> & =\left(\begin{array}{l}
0 \\
0
\end{array}\right)
\end{aligned}
$$

There exists a unique bounded solution $\chi$ of this equation according to proposition 3.1. The function $\psi$ is then given by

$$
\psi=\chi+x\left(|v|^{2}-5\right) M^{\frac{1}{2}}-v_{1}\left(|v|^{2}-5\right) M^{\frac{1}{2}}-v_{1} M^{\frac{1}{2}} .
$$

We follow the same strategy as in part 3.2 to get the iterative solution of (3.9). The first approximate equations for $\tilde{\rho}_{1}:=<\psi M^{\frac{1}{2}}>, \tilde{u}_{1}:=<v_{1} \psi M^{\frac{1}{2}}>, \tilde{\Theta}_{1}:=<\frac{|v|^{2}-3}{2} M^{\frac{1}{2}} \psi>$ are 


$$
\begin{array}{r}
\partial_{x} u_{1}=0 \\
\partial_{x}\left(\Theta_{1}+\rho_{1}\right)=0 \\
\partial_{x}^{2} \Theta_{1}=0
\end{array}
$$

with the bounded solutions $\rho_{1}=\rho_{\infty}^{(1)}, u_{1}=u_{\infty}^{(1)}, \Theta_{1}=\Theta_{\infty}^{(1)}$. Substituting this into (3.9) as in section 3.2 gives the solution

$$
\chi_{1}(x, v)=\left\{\begin{array}{l}
S_{\infty}^{(1)}, \text { if } v_{1}<0 \\
e^{-\frac{x}{v_{1}}}\left(v_{1}\left(|v|^{2}-5\right)+v_{1}\right) M^{\frac{1}{2}}+S_{\infty}^{(1)}\left(1-e^{-\frac{x}{v_{1}}}\right), \text { if } v_{1}>0
\end{array}\right.
$$

with $S_{\infty}^{(1)}=\left(\rho_{\infty}^{(1)}-u_{\infty}^{(1)} v_{1}+\Theta_{\infty}^{(1)} \frac{|v|^{2}-3}{2}\right) M^{\frac{1}{2}} \cdot S_{\infty}^{(1)}$ is determined by

$$
<v_{1}\left(\begin{array}{c}
1 \\
|v|^{2}
\end{array}\right)\left\{\begin{array}{c}
\chi_{1}(0, v) \\
\chi_{1}(\infty, v)
\end{array}\right\} M^{\frac{1}{2}}>=\left(\begin{array}{l}
0 \\
0
\end{array}\right)
$$

The conditions on $\chi_{1}(\infty, v)$ give $u_{\infty}^{(1)}=0$ and $\rho_{\infty}^{(1)}$ and $\Theta_{\infty}^{(1)}$ are determined by the conditions on $\chi_{1}(0, v)$.

Further iteration gives $\chi \sim \chi_{1}+\chi_{2}+\ldots+\chi_{n}$ and

$$
\psi=\chi+\left(x\left(|v|^{2}-5\right)-v_{1}\left(|v|^{2}-5\right)-v_{1}\right) M^{\frac{1}{2}} .
$$

After transforming backward $v \rightarrow-v$ one arrives finally at the iterative solution of (3.9). Using the invariance in $x$ of $\left\langle v_{1} \varphi(x, v) \psi(x, v)>\right.$ and $\left\langle v_{1}^{2} M^{\frac{1}{2}} \varphi(x, v)>\right.$ determines the coefficients $a_{\infty}$ and $c_{\infty}$ in $\varphi(\infty)=\left(a_{\infty}+c_{\infty} \frac{|v|^{2}-3}{2}\right) M^{\frac{1}{2}}$ according to

$$
<v_{1} \varphi(\infty) \psi(\infty)>=<v_{1} \psi(0) \varphi(0)>
$$

and

$$
<v_{1} v_{1} M^{\frac{1}{2}} \varphi(\infty)>=<v_{1} v_{1} M^{\frac{1}{2}} \varphi(0)>
$$

More precisely

$$
\begin{aligned}
a_{\infty}+12 c_{\infty} & =\int_{v_{1}>0} v_{1} k(v) \psi(0, v) d v \\
a_{\infty}+2 c_{\infty} & =\int_{v_{1}>0} v_{1}^{2} k(v) M^{\frac{1}{2}} d v \\
& +\int_{v_{1}<0} v_{1}^{2} \varphi(0, v) M^{\frac{1}{2}} d v
\end{aligned}
$$

For $\varphi(0, v), v_{1}<0$ we substitute again the expression obtained by the Maxwell method. The coefficients are, in this case, determined by the first and third equation in (3.5). 
The value of $\psi_{1}(0, v)$ needed for the first iteration step is calculated as

$$
\psi_{1}(0, v)=\left\{\begin{array}{l}
0, \text { if } v_{1}<0 \\
S_{\infty}^{(1)}+\left(v_{1}\left(|v|^{2}-5\right)+v_{1}\right) M^{\frac{1}{2}}, \text { if } v_{1}>0
\end{array}\right.
$$

with $S_{\infty}^{(1)}=\left(\rho_{\infty}^{(1)}+\Theta_{\infty}^{(1)} \frac{|v|^{2}-3}{2}\right) M^{\frac{1}{2}}$.

\section{Comparison with the variational method:}

Here the variational method can be summarized as follows:

We use the invariance in $x$ of

$$
<v_{1} v_{1} M^{\frac{1}{2}} \varphi(x, v)>\text { and }<v_{1} L^{-1}\left(v_{1}\left(|v|^{2}-5\right) M^{\frac{1}{2}}\right) \varphi>
$$

together with $L^{-1}\left(v_{1}\left(|v|^{2}-5\right) M^{\frac{1}{2}}\right)=v_{1}\left(|v|^{2}-5\right) M^{\frac{1}{2}}$. This yields

$$
<v_{1}^{2} M^{\frac{1}{2}} \varphi(\infty, v)>=<v_{1}^{2} M^{\frac{1}{2}} \varphi(0, v)>
$$

and

$$
<v_{1}^{2}\left(|v|^{2}-5\right) M^{\frac{1}{2}} \varphi(\infty, v)>=<v_{1}^{2}\left(|v|^{2}-5\right) M^{\frac{1}{2}} \varphi(0, v)>.
$$

$\varphi(0, v), v_{1}<0$ is again the reflected density provided by the Maxwell method as above, $\varphi(0, v)=k(v), v_{1}>0$ and $\varphi(\infty, v)=\left(a_{\infty}+c_{\infty} \frac{|v|^{2}-3}{2}\right) M^{\frac{1}{2}}$. This gives the 2 equations

$$
a_{\infty}+2 c_{\infty}=\int_{v_{1}<0} v_{1}^{2} \varphi(0, v) M^{\frac{1}{2}} d v+\int_{v_{1}>0} v_{1}^{2} k(v) M^{\frac{1}{2}} d v
$$

and

$$
10 c_{\infty}=\int_{v_{1}<0} v_{1}^{2}\left(|v|^{2}-5\right) \varphi(0, v) M^{\frac{1}{2}} d v+\int_{v_{1}>0} v_{1}^{2}\left(|v|^{2}-5\right) k(v) M^{\frac{1}{2}} d v
$$

To show that this prescription is equivalent to our method, we observe that equation (3.12) is exactly (3.11). By adding (3.12) to (3.13) the latter is transformed into

$$
\begin{aligned}
a_{\infty}+12 c_{\infty} & =\int_{v_{1}<0} v_{1}^{2} \varphi(0, v)\left(\left(|v|^{2}-5\right)+1\right) M^{\frac{1}{2}} d v \\
& +\int_{v_{1}>0} v_{1}^{2}\left(\left(|v|^{2}-5\right)+1\right) k(v) M^{\frac{1}{2}} d v
\end{aligned}
$$

which is, after some manipulations,

$$
\begin{aligned}
= & \int_{v_{1}>0} v_{1}\left(\rho_{\infty}^{(1)}+\Theta_{\infty}^{(1)} \frac{|v|^{2}-3}{2}\right) M^{\frac{1}{2}} k(v) d v \\
& +\int_{v_{1}>0} v_{1}^{2}\left(\left(|v|^{2}-5\right)+1\right) M^{\frac{1}{2}} k(v) d v \\
= & \int_{v_{1}>0} v_{1} k(v) \psi_{1}(0, v) d v .
\end{aligned}
$$


This is the desired result: indeed this last equation is the same as $(3.10)$ with $\psi(0, v) \sim$ $\psi_{1}(0, v)$ in the first step.

\subsection{Results}

Choosing the incoming function $k(v)=v_{1}\left(|v|^{2}-5\right) M^{\frac{1}{2}}$, one finds that the asymptotic value $c_{\infty}$ is the usual temperature slip coefficient if $u=0$. This value has been computed e.g., by Sone/Onishi [20] as 1.3027 which must again be multiplied by $\sqrt{2}$ to get 1.842 . Other computations and references can be found in Cercignani [6].In particular, we computed the values for $u=0$ :

$\begin{array}{ccrr} & \text { Maxwell } & \text { 1. Step/Variational } & \text { 2. Step } \\ a_{\infty} & -1.567 & -2.059 & -2.098 \\ \frac{c_{\infty}}{2} & 1.567 & 1.821 & 1.839\end{array}$

For $0<u<c$ the values of $a_{\infty}$ and $\frac{c_{\infty}}{2}$ are shown in Figure 4 and 5 .

For $u>c \quad a_{\infty}, b_{\infty}, \frac{c_{\infty}}{2}$ are shown in Figure 6,7,8.

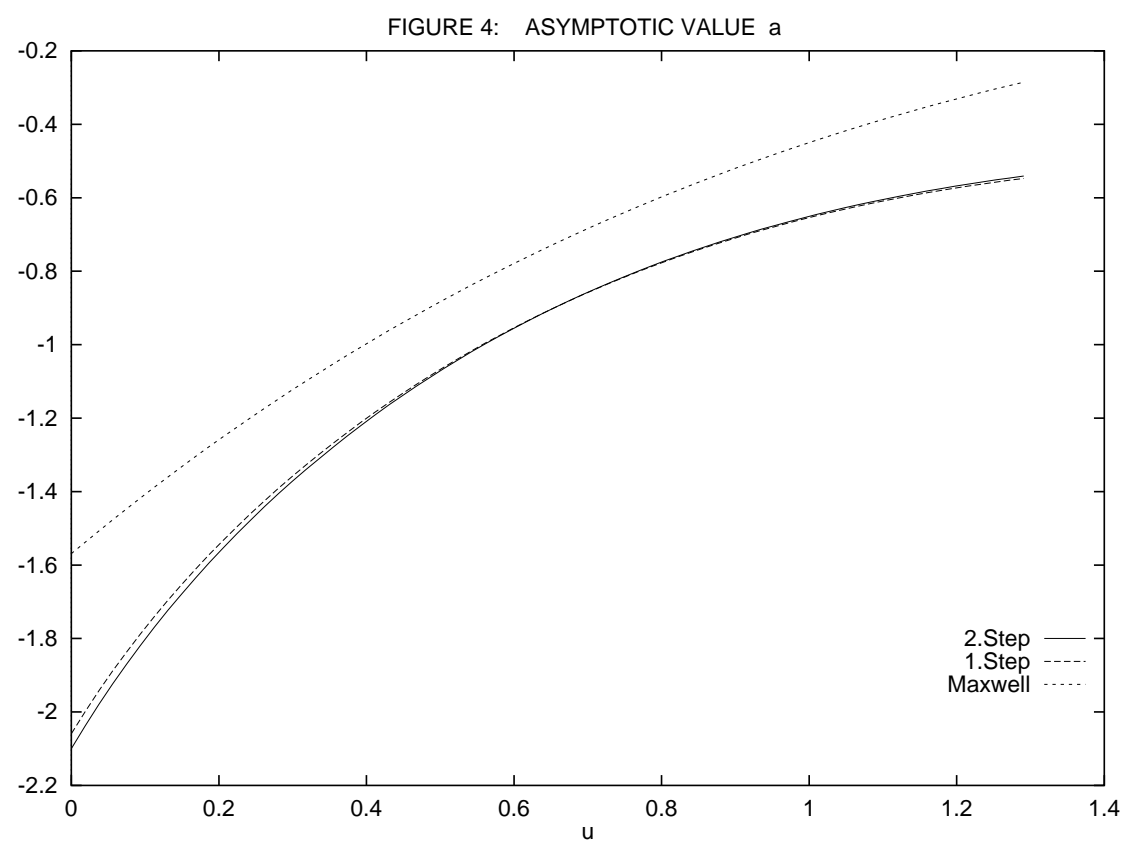


and Cachan/Paris. We wish to thank Profs. C. Bardos, H. Neunzert and R. Illner for their hospitality on these occasions.

\section{References}

[1] K. Aoki, Y. Sone Gas Flows Around the Condensed Phase with Strong Evaporation or Condensation, Advances in Kinetic Theory and Continuum Mechanics, Proceedings of a Symposium in Honor of H. Cabannes, Gatignol, Soubaramayer (Eds.), Springer 1991

[2] M.D. Arthur, C. Cercignani, Nonexistence of a Steady Rarefied Supersonic Flow in a Half Space, ZAMP 31, 634, 1980

[3] J.F. Bourgat, P. Le Tallec, D. Tidriri, Y. Qiu, Numerical Coupling of Nonconservative or Kinetic Models with the Conservative Compressible Navier-Stokes Equations, Fifth International Symposium on Domain Decomposition methods for P.D.E., SIAM, Philadelphia 1991

[4] C. Cercignani, A Variational Principle for Boundary Value Problems, J. of Stat. Phys., Vol 1, No. 2, 1969

[5] C. Cercignani, Mathematical Problems in the Kinetic Theory of Gases, D.C. Pack and H. Neunzert, eds., 129, Lang, Frankfurt, 1980

[6] C. Cercignani, The Boltzmann Equation and its Applications, Springer, 1988

[7] F. Coron, Computation of the Asymptotic States for Linear Halfspace Problems, TTSP 19(2), 89, 1990

[8] F. Coron, F. Golse, C. Sulem, A Classification of Well-posed Kinetic Layer Problems, CPAM, Vol. 41, 409, 1988

[9] F. Golse, Applications of the Boltzmann Equation within the Context of Upper Atmosphere Vehicle Aerodynamics, Computer Meth. in Engineer. and Appl. Mech. Vol. $75,299,1989$

[10] F. Golse, Knudsen Layers from a Computational Viewpoint, TTSP 21 (3), 211, 1992

[11] W. Greenberg, C. van der Mee, V. Protopopescu, Boundary Value Problems in Abstract Kinetic Theory, Birkhäuser, 1987

[12] R. Illner, H. Neunzert, Domain Decomposition: Linking Kinetic and Aerodynamic Descriptions, AGTM preprint 90, Kaiserslautern, 1993

[13] H.G. Kaper, A Constructive Approach to the Solution of a Class of Boundary Value Problems of Mixed Type, J. Math. Anal. Appl. 63, 691, 1978 
[14] A.Klar, Domain Decomposition for Kinetic and Aerodynamic Equations, PhD Thesis, Kaiserslautern, 1994

[15] 1.) S.K. Loyalka, J.H. Ferziger, Model Dependence of the Slip Coefficient, Phys. Fluids 10, No. 8, 1967

2.) S.K. Loyalka, Approximate Method in the Kinetic Theory in Phys. Fluids 11, Vol. 14,1971

[16] A. Lukschin, H. Neunzert, J. Struckmeier, Interim Report for the Hermes Project DPH 6174/91, 1992

[17] J.C. Maxwell, Phil. Trans. Roy. Soc. I, Appendix, 1879; reprinted in The Scientific Papers of J.C.Maxwell, Dover, New York, 1965

[18] E. Ringeisen, PhD Thesis, Paris

[19] 1.) C.E. Siewert, J.R. Thomas, Strong Evaporation into a Half Space I, Z. Angew. Math. Physik, 32, 421, 1981

2.) C.E. Siewert, J.R. Thomas, Strong Evaporation into a Half Space II, Z. Angew. Math. Physik, 33, 202, 1982

[20] Y. Sone, Y. Onishi, Kinetic Theory of Evaporation and Condensation, Hydrodynamic Equation and Slip Boundary Condition, J. Phys. Soc. of Japan, Vol. 44, No. 6, 1981, 1978 